\title{
Antecedentes del oportunismo en las cooperativas agroalimentarias
}

\author{
Jorge Luis Sánchez-Navarro \\ Narciso Arcas-Lario \\ Miguel Hernández-Espallardo
}

RESUMEN: Las cooperativas agroalimentarias son un instrumento con el que cuentan los productores agrarios para afrontar los comportamientos oportunistas de las empresas que les suministran los inputs y de las que adquieren sus productos. Sin embargo, este tipo de comportamientos se reproducen en el interior de la propia organización entre los miembros que interactúan (socios, miembros del Consejo Rector y directivos), originando problemas que afectan de forma negativa al adecuado funcionamiento de las cooperativas y a su competitividad. De aquí la preocupación de los órganos de gobierno de las cooperativas por combatir los comportamientos oportunistas de sus socios, motivo por el que resulta de especial interés conocer los factores o antecedentes que los propician. Precisamente, este es el objetivo de este trabajo empírico, realizado con la información obtenida mediante una encuesta a 140 cooperativas agroalimentarias españolas de primer grado. Los resultados del análisis de los datos obtenidos revelan que mientras la heterogeneidad de los socios, la incertidumbre ambiental y la orientación al mercado de la cooperativa incrementan el oportunismo de los socios; la dependencia de los socios de la cooperativa, la orientación al largo plazo de la relación y la orientación de los socios al mercado lo disminuyen.

PALABRAS CLAVE: Cooperativas agroalimentarias, oportunismo, heterogeneidad, dependencia, incertidumbre, orientación al mercado.

CLAVES ECONLIT: J54, L14, M20, M38, Q13.

Cómo citar este artículo / How to cite this article: SÁNCHEZ-NAVARRO, J.L., ARCAS-LARIO, N. \& HERNÁN-

DEZ-ESPALLARDO, M. (2019): "Antecedentes del oportunismo en las cooperativas agroalimentarias", CIRIEC-

España, Revista de Economía Pública, Social y Cooperativa, 97, 111-136. DOI: 10.7203/CIRIEC-E.97.13282. Correspondencia: Jorge Luis Sánchez Navarro, Universidad Politécnica de Cartagena, jorgel.sanchez@upct.es;

Narciso Arcas Lario, Universidad Politécnica de Cartagena, arcas.lario@upct.es, y Miguel Hernández

Espallardo, Universidad de Murcia, migher@um.es. 


\section{EXPANDED ABSTRACT}

\section{Antecedents of opportunism in Agri-food Cooperatives}

\section{Objectives}

To join an agri-food cooperative is considered an available choice farmers have to deal with manufacturers and food retail chains' opportunistic behaviour. However, opportunism can be observed within the cooperative itself, among its members (partners, Governing Board and managers), which leads to problems that adversely affect cooperatives' performance and competitiveness. The objective of this empirical research is to know the factors that promote cooperative members' opportunism with the ultimate purpose of helping the cooperative's decision makers to hinder these undesirable opportunistic behaviours.

\section{Methodology}

Studies into factors favouring opportunism are scarce in the agri-food cooperatives setting. We found only the work by Marcos et al. (2014), which reported that membership heterogeneity is one such factor. Research is more prominent in the supplier-client relationships setting. Briefly, the meta-analysis by Wang and Yang (2013) revealed that environmental uncertainty and relative dependence promote inter-firm opportunism, while communication, cultural sensitivity, goal congruency and governance reduce it.

We herein study the influence on members' opportunism of members' heterogeneity and dependence, environmental uncertainty, long-term orientation, partners' market orientation and cooperatives' market orientation.

Considering the paper's aims, we propose five hypotheses:

H1: Cooperative members' heterogeneity increases their opportunism.

$\mathrm{H} 2$ : Members' dependence on the cooperative reduces their opportunism.

H3: The environmental uncertainty perceived by members increases their opportunism.

$\mathrm{H} 4$ : The long-term orientation of the members' relationship with the cooperative reduces their opportunism.

H5: The cooperative's market orientation increases members' opportunism.

H6: Members' market orientation reduces their opportunism. 
The data to conduct our empirical research were collected with online surveys sent to key informants of agri-food marketing cooperatives (heads or managing directors). We collected 140 answers.

Regarding the measures, cooperative members' heterogeneity was assessed in terms of members' characteristics (age, level of education, economic status, risk aversion, their objectives in the cooperative, professionalism and technical level), the farm's size, and the products supplied to the cooperative (type, quality and quantity).

Dependence was measured with a single item, assessing members' dependence on the cooperative to fulfil their objectives.

Environmental uncertainty was measured asking about the difficulty to predict the market demand, market tendencies and competitors' actions, along with how often innovations in products arise in the sector, laws and regulations change, and prices vary.

Long-term orientation was measured with a single item, asking whether the cooperative's partners perceive their relationship with the cooperative as a long-term relationship.

Finally, cooperatives' market orientation and partners' market orientation were measured on a semantic differential scale, ranging from "cooperatives/members think that they should focus on producing everything the market demands (market orientation)" to "cooperatives/members think that they should focus on selling everything that is produced (product orientation)".

The measurement of opportunism is based on the definitions and dimensions considered by Wathne and Heide (2000), which classified opportunistic behaviours according to whether behaviour is active or passive, and whether the circumstances surrounding behaviour are new or existing. These authors described four types: evasion, reluctance to adapt, violation and forced renegotiation. The scale asked about members' performing each type of behaviour.

\section{Results}

A tobit regression model was estimated to test the hypotheses.

Our results revealed that partners' heterogeneity $(\beta=0.405)$, environmental uncertainty $(\beta=0.260)$ and cooperatives' market orientation ( $\beta=0.259$ ) increased partners' opportunism, which confirmed $\mathrm{H} 1$, $\mathrm{H} 2$ and $\mathrm{H} 3$. Moreover, partners' dependence on the cooperative $(\beta=-0.277)$, long-term orientation of the relationship $(\beta=-0.252)$ and partners' market orientation $(\beta=-0.246)$ reduced partners' opportunism, which confirmed $\mathrm{H} 4, \mathrm{H} 5$ and $\mathrm{H} 6$. 


\section{Practical conclusion and original value}

The results herein presented are especially interesting from a managerial and an academic viewpoint. They can help cooperatives' decision makers in establishing and implementing mechanisms to combat these undesirable opportunistic behaviours of their memberships. From a theoretical point of view, they help to bridge the gap of empirical studies by analysing opportunism in the agri-food cooperatives setting. Up to this moment, the factors that promote opportunism have been mainly analysed in the field of supplier-customer relationships. However, there are few studies in the literature, especially empirical exploring this issue in the setting of agri-food marketing cooperatives. Most of these organizations implement a monistic management model (both democratic and professional structure is managed by members). Identifying these types of opportunistic behaviour within cooperatives is of a vital importance, since partners themselves can develop opportunistic behaviours both in their relationship as suppliers and as managers. Thus, managing members' opportunism is vital as, otherwise, it has considerable negative consequences on the performance and competitiveness of this type of organizations, challenging the cooperatives' long term viability.

KEYWORDS: Agri-food cooperatives, opportunism, heterogeneity, dependency, uncertainty, market orientation. 


\section{Introducción' 1}

En la actualidad, los productores agrarios cuentan con diversas alternativas para llevar a cabo la comercialización de sus productos. Estas alternativas presentan diferente grado de vinculación entre las partes que intervienen en la relación de intercambio, yendo desde las relaciones de mercado tradicionales, que presentan la menor vinculación, hasta la integración, pasando por formas intermedias basadas en la cooperación (Heide, 1994). Un claro exponente de estas formas intermedias son las cooperativas agroalimentarias, organizaciones que comparten características propias del mercado y de la empresa, motivo por el que son consideradas como una forma híbrida de gobierno de las transacciones (Coque, 2008, Salazar y Galve, 2010).

Se trata de empresas del ámbito de la economía social, con una gran importancia socioeconómica, tanto en términos cuantitativos como cualitativos. En el primer caso, por el elevado número de socios que agrupan, de facturación y de puestos de trabajo que generan. Así, las 3.740 cooperativas agroalimentarias que en 2016 existían en España facturaron 28.993 millones de euros y dieron empleo a 100.831 trabajadores (Cooperativas Agro-alimentarias, 2018). Desde el punto de vista cualitativo, estas organizaciones singulares adquieren un gran protagonismo en el incremento de la competitividad de las empresas de sus socios mediante los servicios que les prestan (formación, asesoramiento, suministros, comercialización, etc.). Estos servicios ayudan a sus socios a mejorar la eficiencia de sus explotaciones, responder a las exigencias del mercado y a protegerse del abuso de poder de negociación y del oportunismo de sus proveedores y clientes (Arcas et al., 2013).

Una de las motivaciones que lleva a los agricultores a incorporarse a cooperativas es hacer frente a los comportamientos oportunistas de las grandes multinacionales que les suministran los inputs para la producción y de las grandes cadenas de distribución e industrias agroalimentarias que se la compran. Sin embargo, estos comportamientos también aparecen en el interior de la propia organización entre los grupos que interaccionan en la misma (socios, miembros del Consejo Rector y directivos) (Harris et al., 1996; lliopoulos y Valentinov, 2012; Bijman et al., 2012).

El oportunismo de estos grupos, que abarca comportamientos tales como engañar, hacer trampas, ocultar información u otras formas más sutiles de violar un acuerdo, aparece en la literatura como una de las debilidades de las cooperativas. Este oportunismo tiene unas consecuencias muy negativas para las organizaciones en general y para las cooperativas agroalimentarias en particular (Marcos et al., 2014). En muchos casos, estas organizaciones apuestan por un modelo monista de gestión, en el que tanto la estructura democrática (distribución del poder de decisión) como la estructura profesional de la

\footnotetext{
1.- Los autores agradecen el apoyo financiero de la Cátedra Cajamar de Cooperativismo Agroalimentario - Universidad Politécnica de Cartagena.
} 
cooperativa (relaciones existentes entre trabajadores y directivos) es gestionada por los propios socios (Garrido et al., 2017), quienes pueden comportarse de forma aprovechada u oportunista. Estos comportamientos provocan los denominados problemas de incentivos (Williamsom, 2004) que afectan de forma negativa al funcionamiento, desempeño y competitividad de las cooperativas. Como consecuencia de estos problemas de incentivos, los socios muestran rechazo a invertir en la cooperativa ante el riesgo de que surjan estos comportamientos oportunistas (Cook y lliopoulus, 2000) y se muestran incapaces de tomar decisiones colectivas de forma eficiente (Iliopoulus y Hendriske, 2009).

Estas consecuencias negativas de los comportamientos oportunistas para las cooperativas provocan que una de las mayores preocupaciones de sus órganos de gobierno sea adoptar las decisiones adecuadas para combatirlos. De aquí, el interés de analizar y comprender los factores 0 antecedentes que propician estos comportamientos indeseables. Sin embargo, a pesar de su importancia, el oportunismo apenas ha sido objeto de estudio en las cooperativas agroalimentarias, siendo escasos los trabajos que se encuentran, sobre todo empíricos (Marcos et al., 2014). En cambio, sí ha sido analizado en el ámbito de las relaciones inter-organizacionales tipo proveedor-cliente (Hansmann's, 1996) y en las alianzas estratégicas (Chiles y McMackin, 1996), apoyándose, en la mayoría de los casos, en las Teorías de los Costes de Transacción (Anderson, 1988; Parkhe 1993; Hill, 1990) de Agencia (John, 1984; Ping, 1993), de los Derechos de Propiedad (Joshi y Stump, 1999) y Relacional (Brown et al., 2000).

En cuanto a los antecedentes del oportunismo de los socios, en la literatura sobre cooperativas agroalimentarias tan solo encontramos evidencia empírica del incremento del mismo conforme aumenta la heterogeneidad de los socios (Marcos et al., 2014). Más numerosos son los trabajos que abordan estos antecedentes en el ámbito de las relaciones proveedor-cliente o vendedor-comprador. Así, encontramos que la dependencia provocada por la inversión en activos específicos reduce la propensión oportunista del que la realiza y la aumenta en su socio (Rokkan et al., 2003). En una línea parecida, se encuentra que existe una relación positiva de las inversiones asimétricas entre las partes (Achrol y Gundlach, 1999). Otros factores como la incertidumbre ambiental (Joshi y Stump, 1999; Lee, 1998; Sako y Helper, 1998; Schilling y Steensma, 2002; Skarmeas et al., 2002) o la congruencia de objetivos entre las partes también han sido considerados por la literatura de relaciones inter-organizacionales (Gundlach et al., 1995; Alchrol y Gundlach, 1999., Brown et al., 2000, Joshi y Stump, 1999; Lai et al., 2005). A modo de síntesis, el meta-análisis realizado por Wang y Yang (2013) revela que la congruencia de objetivos, la sensibilidad cultural, la comunicación, las normas y la gobernanza disminuyen el oportunismo inter-empresarial, mientras que la volatilidad ambiental y la dependencia relativa del fabricante incrementan los comportamientos oportunistas del proveedor.

Considerando la cooperativa agroalimentaria como el resultado de la colaboración entre las empresas agrarias de sus socios, el objetivo de este trabajo es conocer las causas del oportunismo de los mismos. Para ello, en primer lugar se profundiza en el marco teórico que permite conceptualizar el oportunismo e identificar sus causas o antecedentes. A continuación se describe la metodología llevada a cabo en la realización del estudio empírico y se exponen los resultados obtenidos. El trabajo finaliza con las conclusiones y la bibliografía utilizada. 


\section{Fundamentos teóricos}

\subsection{Oportunismo}

El oportunismo puede definirse como "la búsqueda del interés propio con astucia", incluyendo acciones como la mentira, el robo, el engaño y los esfuerzos calculados para confundir a la otra parte o para distorsionar la información (Williamson, 1985). Wathne y Heide (2000) realizan una clasificación del oportunismo en función de si el comportamiento es activo (se hace algo a sabiendas de que no es correcto) o pasivo (no se hace algo a sabiendas de que se debería hacer), y si se da ante circunstancias nuevas o ya existentes en la relación. De esta forma se describen los cuatro tipos de oportunismo que aparecen en la Tabla 1.

\section{Tabla 1. Tipos de oportunismo}

\begin{tabular}{|l|llc|}
\hline \multicolumn{3}{|c|}{ Circunstancia } \\
\hline \multirow{2}{*}{ Comportamiento } & Activo & Renegociación forzosa & Vxistente \\
& Pasivo & Reticencias a de un acuerdo \\
& & Evasión \\
\hline
\end{tabular}

FUENTE: Wathne y Heide (2000).

Evasión: Es el tipo de oportunismo más común en las cooperativas. Aparece cuando un socio no realiza las actividades a las que se había comprometido previamente. Es por tanto un comportamiento oportunista pasivo y sobre circunstancias ya existentes. Un claro ejemplo de evasión puede aparecer cuando el socio no lleva a la práctica en su producción los estándares de calidad que ha acordado con la cooperativa. Como vemos, a priori el socio oportunista obtiene una ventaja a corto plazo al ahorrar costes en la producción, pero tiene un efecto negativo en el largo plazo, tanto para él mismo como para el resto de socios, al empeorar la calidad final de los productos ofrecidos por la cooperativa a sus clientes.

Reticencias a adaptarse: Se refiere a la negativa de un socio a adaptarse ante nuevas exigencias o directrices de la cooperativa por cambios en la demanda de sus clientes. Por tanto, se trata de un oportunismo pasivo y ante circunstancias nuevas. Un ejemplo puede ser la negativa de los socios a introducir en sus cultivos unas determinadas normas de producción sostenible. Se trata de un oportunismo que quizá mejore, o no empeore, la rentabilidad del agricultor en el corto plazo. Pero este comportamiento es realizado en perjuicio de la cooperativa, llevando a una mala adaptación al mercado y a unos peores resultados en el largo plazo. 
Violación de un acuerdo: Este tipo de oportunismo aparece cuando el socio realiza algún acto sabiendo que no debería hacerlo. Es por tanto un oportunismo activo y ante circunstancias ya existentes. Por ejemplo, cuando un socio de una cooperativa en la que se exige exclusividad del abastecimiento, aportando la totalidad del producto, decide desviar una parte para comercializarlo con otros operadores y así obtener un mejor precio que el ofrecido por la cooperativa.

Renegociación forzosa: Se trata de un comportamiento activo ante circunstancias nuevas. Por ejemplo, cuando se produce un cambio de tendencia en el consumo de un producto y los socios que lo están produciendo intentan aprovecharse renegociando con la cooperativa unos precios especiales por el producto aportado.

\subsection{Antecedentes del oportunismo}

\section{Heterogeneidad de los socios}

La heterogeneidad de los socios es uno de los factores que más problemas genera en las cooperativas agroalimentarias (Höhler y Kühl, 2017). A medida que los miembros de las cooperativas son más diversos en términos de tamaño de las explotaciones, tipos de productos, edad, objetivos perseguidos 0 aversión al riesgo, los problemas en este tipo de organizaciones se agravan (Kyriakopoulos, 1998; Bijman y Hendrikse, 2002). En este sentido, Marcos et al. (2014) confirman que ciertos tipos de heterogeneidad de los socios están relacionados negativamente con la disposición de los mismos a invertir en la cooperativa, lo que según la clasificación anterior corresponde al tipo de comportamiento oportunista de "evasión". Por su parte, Gripsrud et al. (2000) y Kalogeras et al. (2009) sostienen que la diversidad de objetivos que podemos encontrar en una base social heterogénea hacen que los objetivos de la cooperativa no estén bien definidos, aumentando así la discrecionalidad de los miembros para tomar decisiones que les beneficien a ellos en detrimento del interés colectivo. De forma similar, Carson et al. (2004) argumentan que esta heterogeneidad hace necesario debatir y renegociar continuamente los acuerdos tomados, lo que se traduce en numerosas confrontaciones entre los miembros y mayor oportunidad para adoptar comportamientos que buscan el interés propio, esto es, comportamientos oportunistas. Así, de cara a una renegociación, los socios con mayores inversiones específicas en la cooperativa están en desventaja debido a su deseo de continuar en ella, ya que estas inversiones actúan como barrera de salida y, por tanto, son más vulnerables al oportunismo de otros socios que muestran reticencias a invertir, aunque vaya en contra del interés general (Hart y Moore, 1990). Además, la heterogeneidad también provoca que el control de los socios se vuelva más problemático, al tratarse de una base social más diversa, lo que se presenta como un ambiente más favorable para la puesta en práctica de comportamientos oportunistas (Fulton y Giannakas, 2001). A la vista de las aportaciones anteriores se plantea la siguiente hipótesis:

H1: La heterogeneidad de los socios de la cooperativa tiene un efecto positivo sobre su oportunismo. 


\section{Dependencia}

La investigación pionera sobre la dependencia se debe a Emerson (1962), quien argumenta que la dependencia de A sobre B es directamente proporcional al grado en que A necesita a B para conseguir sus objetivos, e inversamente proporcional a la disponibilidad de relaciones alternativas. En esta misma línea, para Frazier (1983) la dependencia es la necesidad que tiene una empresa de mantener la relación con otra para conseguir sus objetivos. En el caso de las cooperativas, no se encuentran estudios que analicen la dependencia como antecedente del oportunismo. Sin embargo, si aparecen algunos en el ámbito de las alianzas estratégicas. En este sentido, Das y Rahman (2010) sostienen que, si el grado de dependencia del socio con la alianza es bajo, el primero será más propenso a comportarse de forma oportunista ya que puede cambiar más fácilmente de alianza o socios. En el ámbito de las relaciones proveedor-cliente, Hawkins et al. (2008) sostienen que es el constructo que afecta al oportunismo por excelencia, argumentando que la inversión de una parte en una relación de intercambio en activos específicos, un elemento que aumenta su dependencia de la relación, reduce la propensión del inversor a comportarse de forma oportunista, pero simultáneamente aumenta el riesgo de sufrir los comportamientos oportunistas de la otra parte (Hawkins et al, 2008). De forma similar, Joshi y Stump (1999) argumentan que la dependencia del fabricante con respecto al proveedor está negativamente relacionada con sus comportamientos oportunistas. Teniendo en cuenta lo anterior, en el ámbito de las cooperativas agroalimentarias se espera que una mayor dependencia del socio con la cooperativa (entendida como una necesidad de la misma para conseguir sus objetivos) disminuirá la adopción de comportamientos oportunistas. Esto es así por el miedo a ser expulsado de la relación, pues en el caso de producirse, supondría, a elevados niveles de dependencia, un gran perjuicio económico para el socio. En vistas a esto, proponemos la siguiente hipótesis:

H2: La dependencia de los socios de la cooperativa tiene un efecto negativo sobre su oportunismo.

\section{Incertidumbre ambiental}

La incertidumbre se refiere a la falta de seguridad, de confianza o de certeza sobre algo, especialmente cuando ese algo crea inquietud. Según Sako y Helper (1998) puede ser de dos clases, comportamental 0 ambiental. La primera tiene su origen en el comportamiento de los individuos. La segunda, en la que nos centramos en este trabajo, encuentra su origen más allá del comportamiento de los individuos, pues se refiere a la falta de seguridad o control sobre los comportamientos o tendencias que experimenta el mercado, lo que la convierte en algo totalmente exógeno (Sako y Helper, 1998).

La incertidumbre ha sido calificada como uno de los antecedentes del oportunismo en las cooperativas (Harris et al, 1996). Concretamente, en aquellas cooperativas en las que existe un compromiso para aceptar toda la producción de los miembros, estos tienen incentivos para eludir la calidad de los productos que entregan a la cooperativa (comportamiento oportunista). Además, el problema se agrava 
cuando hay variaciones en los precios de los productos o en la calidad de los mismos, es decir, cuando la incertidumbre ambiental es mayor (Harris et al, 1996). En el ámbito de las alianzas estratégicas, Lee (1998) comprobó que la incertidumbre en la toma de decisiones de los individuos es un antecedente del oportunismo y Sako y Helper (1998) encuentran que la incertidumbre en el mercado y el desarrollo tecnológico son antecedentes de la percepción de comportamientos oportunistas que el proveedor tiene sobre el cliente.

Centrándonos en las relaciones proveedor-cliente, Hawkins et al. (2008) afirman que los investigadores se han centrado, sobre todo, en el análisis de la incertidumbre ambiental como antecedente del oportunismo, encontrándose, generalmente, una relación positiva (Joshi y Stump, 1999; Lee, 1998; Sako y Helper, 1998; Schilling y Steensma, 2002; Walker y Weber, 1984). En ámbitos con poca o ninguna incertidumbre, podrían contemplarse todas (o casi todas) las contingencias que pueden surgir en la relación, lo que permitiría que el contrato cumpliera adecuadamente una función de salvaguarda del oportunismo de cualquiera de las partes (Walker y Weber, 1984). En la misma línea, Luo (2007) encuentra que el nivel de oportunismo exhibido por la empresa en su conjunto se debe, en parte, a la incertidumbre ambiental. Con base en las aportaciones anteriores se plantea la siguiente hipótesis:

H3: La incertidumbre ambiental percibida tiene un efecto positivo sobre el oportunismo de los socios.

\section{Orientación a largo plazo de la relación de los socios con la cooperativa}

Uno de los determinantes que lleva a las organizaciones a establecer relaciones a largo plazo es la búsqueda de la estabilidad que les permita mejorar su eficiencia (Oliver, 1990). De aquí el interés de analizar la "orientación a largo plazo" de las relaciones, entendida como una predisposición o actitud a establecer y mantener relaciones duraderas (Ganesan, 1994; Swan et al., 1999), y en qué medida la misma afecta al oportunismo de las partes. Sin embargo, este posible antecedente del oportunismo no ha sido analizado en el ámbito de las cooperativas, y tampoco se encuentran trabajos empíricos que contrasten sus efectos en las alianzas estratégicas. Desde una perspectiva teórica, Das (2006) plantea que, en las alianzas estratégicas, una orientación de la relación a largo plazo de una de las partes se relaciona con un bajo potencial de comportamientos oportunistas, tanto por temor a las posibles repercusiones futuras como por el hecho de que desigualdades puntuales pueden ser compensadas en un futuro. De forma similar, en las relaciones proveedor-cliente, Heide y John (1990) argumentan que la interacción futura entre las partes ofrece la oportunidad de recompensar el buen comportamiento y castigar el oportunismo. En esta misma línea, Hawkins et al. (2008) sostienen que el oportunismo se reduce con expectativas de relaciones a largo plazo y Joshi y Stump (1999) que los proveedores con elevadas normas relacionales desarrollan relaciones a largo plazo que, a su vez, incrementan el compromiso y reducen el oportunismo del cliente. A la vista de todas las aportaciones anteriores y siguiendo la idea de Das (2006) para el caso de las alianzas estratégicas, en el ámbito de las cooperativas agroalimentarias se espera que los socios con una elevada predisposición a establecer relaciones duraderas con la cooperativa encuentren menos incentivos para comportarse de 
forma oportunista. Tanto por el temor a posibles sanciones que puedan incluir la expulsión o, al menos, el deterioro de la relación con la dirección de la cooperativa, como por el hecho de que, si se pretende permanecer en la cooperativa durante un horizonte temporal de largo plazo, existirá la posibilidad de compensar, en el futuro, los beneficios potenciales que un comportamiento oportunista les podría brindar en el corto plazo.

Teniendo en cuenta todo lo anterior se propone la siguiente hipótesis:

H4: La orientación a largo plazo de la relación de los socios con la cooperativa tiene un efecto negativo sobre su oportunismo.

\section{Predisposición a la orientación al mercado de la cooperativa y de los socios}

Estar orientado al mercado significa innovar y adaptar la organización a las demandas y circunstancias del mercado (Baker y Sinkula, 1999). Según Arcas (2000), la orientación al mercado de las cooperativas agrarias puede ser considerada como un recurso intangible o habilidad superior que tienen para conocer las necesidades del mercado, diseminarlas por toda la organización y ofrecer una respuesta que las satisfaga en forma de una oferta de valor superior. De esta forma, las cooperativas orientadas al mercado adoptan una perspectiva a medio y largo plazo, averiguando el producto que exige el mercado, comunicándolo a sus socios y restringiendo la libertad de estos con el fin de que produzcan las calidades y cantidades que demanda el mercado. Posteriormente, las empresas agrarias actúan de forma coordinada con sus cooperativas para llevar a cabo la respuesta a las necesidades del mercado mediante una oferta de valor para este.

La orientación al mercado es reconocida como un importante factor de desempeño de las cooperativas agroalimentarias (Kyriakopoulos et al., 2004). Sin embargo, las especiales características de estas organizaciones, en las que los socios, además de propietarios, son también proveedores y clientes de la cooperativa, hace que su orientación al mercado se enfrente con una cierta problemática debido a la necesidad de la cooperativa de satisfacer a dos tipos de clientes. Por un lado, están los clientes externos, intermediarios (cadenas detallistas de distribución) y finales (consumidores), que la cooperativa tiene en el mercado y que abastece con sus productos. Y, por otro, están los socios, clientes internos de la cooperativa que se incorporan a ella para que esta les preste los servicios dirigidos a satisfacer sus necesidades relacionadas, entre otras, con la comercialización de los productos que aportan como proveedores, y cuya permanencia en la cooperativa es fundamental para la supervivencia de esta como empresa. De aquí el interés de que la cooperativa concilie ambas orientaciones: a los clientes externos (orientación al mercado) y a los internos (orientación al socio o a la producción). Con la orientación al mercado, la cooperativa da prioridad a la generación de valor percibido por parte de su público objetivo, clientes externos (Lajara y Server, 2016) mientras que, con la orientación a la producción, la cooperativa se enfoca a prestar un servicio de comercialización de toda la producción de sus socios en las mejores condiciones posibles. 
La predisposición a la orientación al mercado (o a la producción) de la cooperativa podría entenderse que es un reflejo de la predisposición a la orientación al mercado (o a la producción) de sus socios, ya que este aspecto compete al Consejo Rector de la misma, cuyos miembros son elegidos de forma democrática por los socios en la Asamblea General. Sin embargo, aunque cabe pensar que los socios elegirán a los miembros del Consejo Rector cuyos ideales estén en línea con los del propio socio, esto no implica que una vez estos hayan sido elegidos decidan adoptar medidas dirigidas a mejorar la competitividad de la cooperativa, entre ellas su orientación al mercado (por ejemplo, incentivando entre sus agricultores el cultivo de una nueva variedad muy valorada en el mercado, o no comercializando los productos que no respondan a unos estándares mínimos de calidad), con el fin de beneficiar en última instancia a los socios.

En base a los argumentos anteriores pensamos que, en un aspecto de gestión, como es la orientación al mercado de la cooperativa, esta no siempre estará alineada con la orientación de los socios. Por ello, se ha considerado por un lado la orientación al mercado de la cooperativa, y por otro la de los socios.

La orientación al mercado apenas ha sido objeto de estudio en el ámbito de las cooperativas agroalimentarias (Benos et al., 2016; Sisay et al., 2017). En particular, no encontramos ningún trabajo que analice los vínculos entre la orientación al mercado de las partes que intervienen en una relación y la adopción de comportamientos oportunistas. No obstante, en el ámbito de las relaciones sociocooperativas agroalimentarias, se podría esperar una relación positiva entre la orientación al mercado de las cooperativas y el oportunismo de sus socios. Ello obedece a que, una mayor orientación al mercado, en detrimento de la orientación a la producción, podría producir que parte de la base social perciba que la atención de la cooperativa se aleja de los socios, colocándolos a estos en una posición subordinada, pues el socio pasa de ser el fin último de la cooperativa, a ser un instrumento para alcanzar los objetivos de la misma, lo que puede crear un sentimiento de lejanía con la cooperativa y traducirse en la adopción de comportamientos oportunistas. Teniendo en cuenta lo anterior se plantea la siguiente hipótesis:

H5: La predisposición a la orientación al mercado de la cooperativa tiene un efecto positivo en el oportunismo de sus socios.

Respecto a la orientación al mercado de los socios, se pueden realizar dos apreciaciones. De un lado, puesto que los socios orientados al mercado dan prioridad a la generación de valor percibido por sus clientes, cabe pensar que adoptarán comportamientos alejados del oportunismo que permitan una mayor generación de valor para el mercado. Por otra parte, las cooperativas agroalimentarias están en un eslabón más avanzado en la cadena agroalimentaria que sus socios. Esto hace que las primeras muestren un mayor sesgo a orientarse al mercado y que asuman, de hecho, una cierta responsabilidad en promover dicha orientación también en sus socios (Arcas y Hernández, 2003). Por lo tanto, si la relación que mantienen con la cooperativa puede contribuir a mejorar su deseada orientación al mercado, cabe pensar que no llevarán a cabo comportamientos que pudieran poner en peli- 
gro su continuidad en la cooperativa. Por ello, también de forma exploratoria, se propone la siguiente hipótesis:

H6: La predisposición a la orientación al mercado de los socios de la cooperativa tiene un efecto negativo en el oportunismo de los mismos.

\section{Metodología del estudio empírico}

\subsection{Población y recogida de información}

La población objeto de estudio en este trabajo está formada por las cooperativas agroalimentarias españolas de primer grado. La misma la forman unas 3.142 cooperativas (Cooperativas Agro-alimentarias, 2018). Para identificar los componentes de esta población, nos ayudamos del directorio que provee Cooperativas Agro-alimentarias, la organización nacional que representa al 70\% del total de cooperativas.

Los datos para abordar el estudio se obtuvieron mediante una encuesta online dirigida al informante clave de la cooperativa (gerente o responsable de la gestión de la cooperativa). Se consiguieron un total de 140 encuestas válidas, configurando las cooperativas que las respondieron la muestra de este estudio. En el cuestionario se incluyeron algunas preguntas que ayudan a caracterizar a estas cooperativas. Cabe señalar que tienen una media de 390 socios y una facturación media de 7,17 millones de euros. Además, el 24,29\% comercializa frutas u hortalizas, el 30,71\% aceite, el 7,14\% cítricos, el $31,43 \%$ vino y un $6,43 \%$ comercializa ganadería y/o productos cárnicos.

\subsection{Desarrollo de medidas}

Para medir el oportunismo y algunos de los conceptos considerados como antecedentes del mismo, se han utilizado escalas multi-item de clasificación por categorías de 7 puntos. Estas son congruentes con la conceptuación que se ha realizado en el apartado 2 y han sido adaptadas a las particularidades de las cooperativas de este trabajo. Esta forma de proceder nos aproxima a la validez de contenido -el instrumento de medida recoge todo el significado o contenido del concepto-. Sin embargo, otros han sido medidos utilizando un único ítem, pues como argumenta Wanous et al. (1997), "si el constructo a medir está suficientemente acotado o no es ambiguo para el que responde, entonces una medida de un único ítem es suficiente". 
Para determinar la bondad de las escalas se ha utilizado como criterio de fiabilidad el coeficiente Alpha de Cronbach. Churchill (1979) expone que, para las primeras etapas de cualquier investigación, valores del coeficiente entre 0,5 y 0,6 puede ser suficientes para garantizar la fiabilidad de las escalas.

La medición del oportunismo se basa en la definición y dimensiones consideradas por Watne y Heide (2000) (Tabla 2). En este sentido, el cuestionario utilizado recoge una serie de ítems que se corresponden con cada uno de los cuatro tipos de comportamientos oportunistas que recoge la Tabla 1.

Con respecto a los antecedentes del oportunismo, en lo que se refiere a la heterogeneidad, la literatura no recoge medidas al respecto. Aunque algunos autores, sí han hecho sugerencias sobre los aspectos que se tienen que considerar para medir este factor (lliopoulus y Cook, 1999; Österberg y Nilsson, 2009). Por tanto, siguiendo estas sugerencias, la heterogeneidad se ha medido con 12 ítems (Tabla 3).

\section{Tabla 2. Escala de medida del oportunismo}

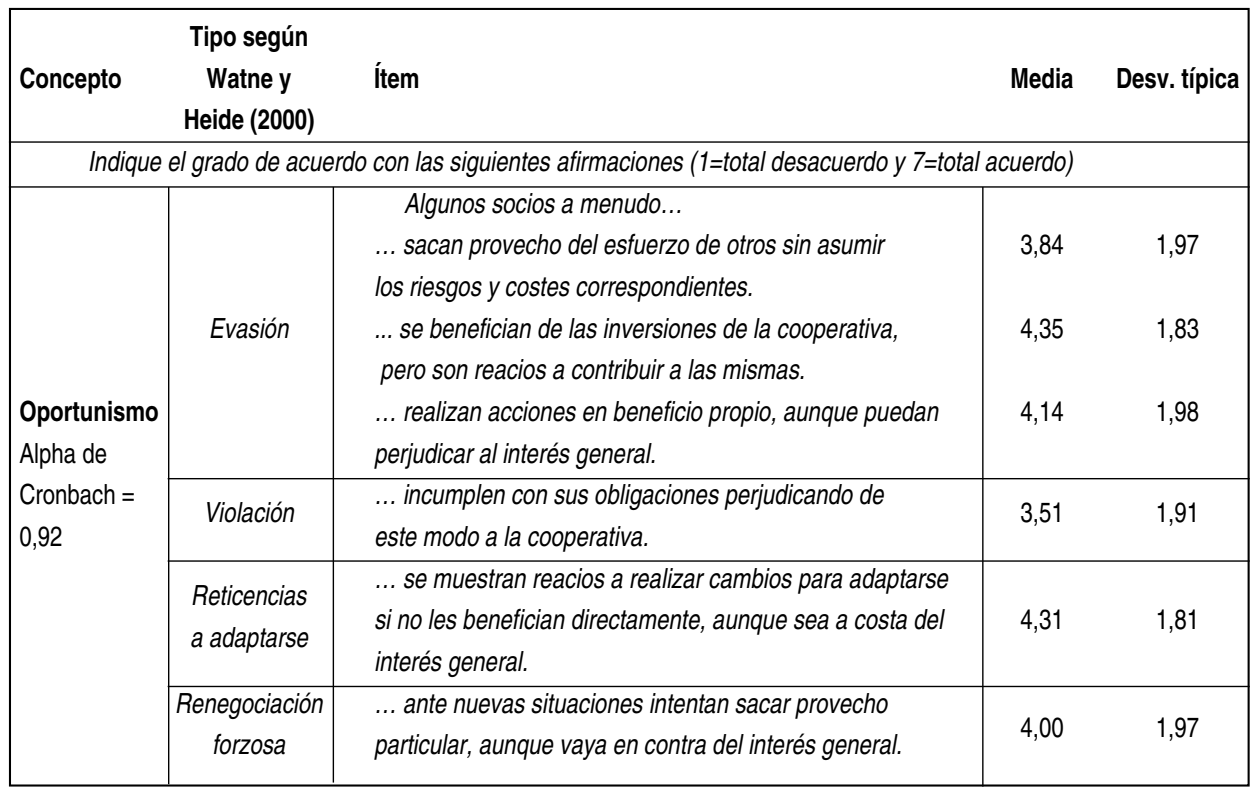




\section{Tabla 3. Escala de medida de la heterogeneidad de los socios}

\begin{tabular}{|c|c|c|c|}
\hline Concepto & Ítem & Media & Desv. típica \\
\hline \multicolumn{4}{|c|}{$\begin{array}{l}\text { Indique en qué medida el conjunto de los socios de su cooperativa son parecidos entre sí (muy homogéneos) con respecto } \\
\text { a... (siendo, } 1=\text { =muy diferentes y } 7=\text { muy parecidos) }{ }^{a} \text {. }\end{array}$} \\
\hline & Tamaño de las explotaciones. & 3,96 & 1,51 \\
\hline & Edad & 3,86 & 1,29 \\
\hline & Grado de formación & 3,52 & 1,44 \\
\hline & Nivel económico & 3,64 & 1,34 \\
\hline & Tipos de productos que suministran. & 1,99 & 1,46 \\
\hline Heterogeneidad & Calidad de los productos que suministran. & 2,55 & 1,25 \\
\hline Alpha de & Cantidad de sus aportaciones a la cooperativa. & 3,78 & 1,82 \\
\hline \multirow[t]{5}{*}{ Cronbach $=0,79$} & Profesionalidad o nivel técnico. & 3,51 & 1,33 \\
\hline & Percepción sobre la importancia que tiene la orientación de la cooperativa al mercado. & 3,71 & 1,50 \\
\hline & Objetivos que persiguen dentro de la cooperativa & 2,79 & 1,52 \\
\hline & Grado de dependencia de la cooperativa para comercializar sus productos. & 2,31 & 1,43 \\
\hline & Por lo tanto entre 1 y 7 , el grado de homogeneidad de sus socios & 3,35 & 1,06 \\
\hline
\end{tabular}

a La medida utilizada es de homogeneidad (a mayor valor, más homogéneos son los socios). Como el concepto objeto de estudio es la heterogeneidad, para la obtención de los descriptivos presentados en la tabla, así como en los análisis posteriores, las puntuaciones han sido invertidas utilizando la regla 8 - valoración del ítem.

La dependencia se ha medido utilizando un único ítem, en el que el entrevistado manifiesta su grado de acuerdo con la afirmación: Sus ingresos dependen exclusivamente de sus ventas a través de la cooperativa. Tras el análisis de los datos, encontramos que este factor tiene una media de 3,87 y una desviación típica de 1,87.

Para medir la incertidumbre ambiental, siguiendo a Achrol y Stern (1988), se utilizan 6 ítems (Tabla 4). 


\section{Tabla 4. Escala de medida de la incertidumbre ambiental}

\begin{tabular}{|l|l|rc|}
\hline Concepto & \multicolumn{2}{c|}{ Ítem } & Media Desv. típica \\
\hline \multicolumn{2}{|c|}{ Indique el grado de acuerdo con las siguientes afirmaciones (1=total desacuerdo y 7=total acuerdo). } \\
\hline \multirow{3}{*}{ Incertidumbre } & Es muy difícil predecir los volúmenes de producción que demandará el mercado. & 4,85 & 1,67 \\
Ambiental & Es muy difícil predecir las tendencias del mercado. & 5,00 & 1,61 \\
Alpha de & Es muy difícil predecir cuáles van a ser las acciones de la competencia & 4,48 & 1,51 \\
Cronbach =0,69 & En el sector surgen frecuentemente innovaciones en los productos. & 3,83 & 1,73 \\
& Los precios de venta de nuestros productos son muy cambiantes. & 4,81 & 1,87 \\
& La legislación y reglamentos que nos afectan cambian a menudo. & 4,34 & 1,77 \\
\hline
\end{tabular}

La orientación a largo plazo de la relación socio-cooperativa se ha medido con un único ítem en el que el entrevistado manifiesta su grado de acuerdo con la afirmación: Ven su relación con la cooperativa como una relación a largo plazo. Tras el análisis de los datos, encontramos que este factor tiene una media de 4,97 y una desviación típica de 1,73.

Para medir la predisposición a la orientación al mercado de la cooperativa se toma el valor que los encuestados adjudican a la siguiente cuestión: 1= La cooperativa piensa que debe centrarse en producir lo que el mercado demande (orientación al mercado) a $7=$ La cooperativa piensa que debe centrarse en vender todo lo que produzcan sus socios (orientación a la producción). Como se puede observar, a un mayor valor del ítem corresponde una mayor orientación a la producción de la cooperativa en detrimento de la orientación al mercado. Sin embargo, dado que el concepto utilizado en el estudio es la orientación al mercado, para la obtención de la media $(4,00)$ y la desviación típica $(1,94)$, así como en los análisis posteriores, se han invertido las puntuaciones utilizando la regla 8 - valoración del ítem. De esta forma la variable utilizada mide la primacía de la orientación al mercado sobre la orientación a la producción de la cooperativa.

Por último, para medir la predisposición a la orientación al mercado de los socios se ha utilizado la siguiente escala: $1=$ Los socios de la cooperativa piensan que tienen que producir lo que el mercado demande (orientación al mercado) a 7= Los socios de la cooperativa piensan que tienen que centrarse en vender todo lo que produzcan (orientación a la producción). Por la misma razón que en el caso anterior y utilizando la misma regla, las puntuaciones han sido invertidas obteniéndose así una variable que mide la primacía de la orientación al mercado de los socios sobre la orientación a la producción. En este caso se ha obtenido una media de 3,17 y una desviación típica de 1,74. 


\section{Resultados}

Para contrastar las hipótesis planteadas se lleva a cabo la estimación del modelo tobit que a continuación se detalla. El carácter censurado de la variable dependiente (a 1 y 7 ), implica que sus valores no sigan una distribución normal ${ }^{2}$, de modo que la estimación de los parámetros mediante el método estadístico de mínimos cuadrados ordinarios (MCO) llevaría a resultados sesgados e inconsistentes (Greene, 1997):

$$
\begin{aligned}
& \mathrm{OPO}_{i}=\beta_{\mathrm{O}}+\sum_{i=1}^{n} x_{i}+\beta_{1} \mathrm{HET}_{i}+\beta_{2} \mathrm{DEP}_{i}+\beta_{3} \mathrm{IA}_{i}+\beta_{4} \mathrm{OLP}_{i}+\beta_{5} \text { OMCOOP }_{i} \\
& +\beta_{6} \text { OMSOC }_{i}+\varepsilon_{i}
\end{aligned}
$$

Donde:

OPO: oportunismo.

$\sum_{i=1}^{n} x_{i}$ : conjunto de variables control.

HET: heterogeneidad.

$D E P$ : dependencia.

IA: incertidumbre ambiental.

OLP: orientación a largo plazo.

OMCOOP: predisposición a la orientación al mercado de la cooperativa.

OMSOC: predisposición a la orientación al mercado del socio.

$\varepsilon$ : término de error.

Los resultados obtenidos de la estimación del modelo se recogen en la Tabla 5.

2.- Se ha realizado la prueba de normalidad (Skewness-Kurtosis) en Stata basada en el coeficiente de asimetría de la distribución de probabilidad de una variable y el coeficiente de curtosis, de forma conjunta. Para nuestra variable dependiente (Oportunismo) obtenemos un valor del estadístico Chi2 de 15.17 ( $p$-valor =0,0005) por lo que rechazamos la hipótesis nula de normalidad. 


\section{Tabla 5. Resultados del modelo de regresión del oportunismo de los socios como variable dependiente}

\begin{tabular}{|c|c|c|c|}
\hline Variables independientes & Coeficiente & Valort & Error estándar \\
\hline Heterogeneidad & $0,405^{* *}$ & 2,49 & 0,163 \\
\hline Dependencia & $-0,277^{\star * *}$ & $-3,86$ & 0,072 \\
\hline Incertidumbre ambiental & 0,260 ** & 2,26 & 0,115 \\
\hline Orientación de la relación a largo plazo & $-0,252^{* * *}$ & $-3,40$ & 0,074 \\
\hline Orientación al mercado de la cooperativa & $0,259^{\star \star *}$ & 3,71 & 0,070 \\
\hline Orientación al mercado del socio & $-0,246^{\star \star \star}$ & $-3,08$ & 0,080 \\
\hline \multicolumn{4}{|l|}{ Variables de control } \\
\hline \multicolumn{4}{|l|}{ Tipo de producto que comercializa } \\
\hline Hortalizas & $-0,354$ & $-0,83$ & 0,424 \\
\hline Fruta & $-0,510$ & $-1,37$ & 0,370 \\
\hline Cítricos & 0,084 & 0,16 & 0,509 \\
\hline Vino & $-0,528^{*}$ & $-1,94$ & 0,272 \\
\hline Aceite & $-0,243$ & $-0,91$ & 0,267 \\
\hline Leche y/o derivados lácteos & $-0,157$ & $-0,35$ & 0,446 \\
\hline Ganado y/o productos cárnicos & $-0,334$ & $-0,65$ & 0,512 \\
\hline Otros & 0,173 & 0,62 & 0,281 \\
\hline Número de socios & $-0,001$ & $-1,03$ & 0,001 \\
\hline \multicolumn{4}{|l|}{ Valor Chi² (significación) $\quad 70,41(0,000)$} \\
\hline \multicolumn{4}{|l|}{$\begin{array}{ll}P \text {-seudo } R^{2} & 0,132\end{array}$} \\
\hline
\end{tabular}

${ }^{*} p<0,10 ;{ }^{* *} p<0,05 ;{ }^{* *} p<0,01$

Como se puede observar, obtenemos un P-seudo $R^{2}$ de 0,132 . Además, en base a los resultados se pueden aceptar las hipótesis planteadas, observándose que la heterogeneidad de los socios $(\mathrm{H} 1)$, la incertidumbre ambiental $(\mathrm{H} 3)$, y la orientación al mercado de la cooperativa $(\mathrm{H} 5)^{3}$ tienen un efecto positivo sobre el oportunismo de los socios. Por su parte, tal y como se postuló, se ha encontrado que la dependencia del socio $(\mathrm{H} 2)$, su orientación a largo plazo $(\mathrm{H} 4)$ y su orientación al mercado (H6) reducen el oportunismo de los socios de las cooperativas.

3.- Para contrastar esta hipótesis, dado que los argumentos empleados para su justificación pueden presumir una orientación a la producción de los socios, se ha estimado el modelo planteado para los elementos de la muestra que presenta una clara orientación a la producción. El criterio utilizado para determinar esta orientación a la producción ha sido la media de la variable OMSOC más su desviación típica, de forma que $\forall i \in N$ si OMSOC $i<x_{\text {omsoc }}+\sigma_{\text {omsoc }}$ se considera que el elemento $i$ está orientado a la producción. El número de elementos orientados a la producción asciende a 110. Los resultados de esta estimación revelan que $\beta_{5}=0.300^{* * * * *}$ (valor $t=4,39$ ), lo que nos permite aceptar H5. 


\section{Conclusiones}

Las cooperativas agroalimentarias son una de las alternativas con las que cuentan los productores agrarios para hacer frente a los comportamientos oportunistas de sus proveedores y clientes. Sin embargo, esto no evita la presencia de oportunismo, ya que este tipo de comportamientos también se observan en el interior de la propia organización, originando problemas que afectan de forma negativa a la disposición de sus socios a invertir y al funcionamiento, desempeño y competitividad de la cooperativa. Por ello, una de las mayores preocupaciones de sus órganos de gobierno es adoptar las decisiones para combatir este tipo de comportamientos, motivo por el que resulta de especial interés conocer los factores o antecedentes que los propician. Precisamente, este es el objetivo de este trabajo empírico, realizado con la información obtenida mediante una encuesta a 140 cooperativas agroalimentarias españolas.

Los resultados obtenidos evidencian que la heterogeneidad de los socios de las cooperativas agroalimentarias incrementa el oportunismo de los mismos. Factores como la heterogeneidad en edad, tamaño de sus explotaciones, tipos de productos que aportan, objetivos que persiguen, 0 aversión al riesgo, hacen que la definición de los objetivos a conseguir por la cooperativa se convierta en una difícil tarea de cara a satisfacer los intereses de todos los socios. Sobre todo, cuando se intentan establecer objetivos en una o pocas líneas de actuación, pues los socios menos empatizados con esta línea pueden incrementar su discrecionalidad para tomar decisiones que les beneficien a ellos en detrimento del interés colectivo.

En contraste con lo anterior, se ha encontrado evidencia empírica del efecto negativo que sobre el oportunismo de los socios tiene la dependencia que tienen de la cooperativa y de la orientación a largo plazo de la relación que mantienen con ella. Conforme los socios son más dependientes de la cooperativa, en mayor medida la necesitan para conseguir sus objetivos y, por lo tanto, cabe pensar que estos evitarán comportarse de forma oportunista por miedo a las repercusiones que ello podría tener, incluida su expulsión. Además, si el socio tiene intención de mantener la relación con la cooperativa en el largo plazo, tendrá menos incentivos para comportarse de forma oportunista para compensar posibles pérdidas o desequilibrios. El mayor horizonte temporal de la relación le permitirá compensarlos en el futuro sin necesidad de actuar de mala fe persiguiendo su interés propio y perjudicando al resto de socios.

El oportunismo también se ve afectado por la incertidumbre ambiental. Concretamente, si la cooperativa carece de control sobre los comportamientos o tendencias que experimenta el mercado, no podrá establecer contratos que contemplen todas o casi todas las contingencias que podrían surgir en la relación, dando así la oportunidad a los socios para realizar actos con los que aprovecharse ante 
este tipo de situaciones. Dado el carácter exógeno de esta incertidumbre ambiental, poco pueden hacer los órganos de gobierno de la cooperativa al respecto.

Por último, el efecto de la orientación al mercado sobre el oportunismo de los socios presenta distinto signo según se considere la orientación al mercado de la cooperativa o la de sus socios. Los resultados muestran que mientras la orientación al mercado de la cooperativa incrementa el oportunismo, la de los socios lo reduce. Debido a que las cooperativas han de satisfacer a dos tipos de clientes, los externos (cadenas de distribución y consumidores finales) y los internos (socios), la decisión de orientarse en mayor medida al mercado o a la producción resulta problemática. Si la cooperativa aboga por una orientación al mercado, los socios podrían percibir que son un mero instrumento para alcanzar los objetivos de la misma, lo que puede generar un sentimiento de lejanía con la cooperativa y propiciar comportamientos oportunistas de los socios. A pesar de este hallazgo, existe un consenso de que las cooperativas agroalimentarias deben estar orientadas al mercado por ser un elemento clave para mejorar su competitividad (Lajara y Server, 2016) No obstante, las cooperativas tienen que asegurarse de que los socios perciben los beneficios que ello conlleva (Kyriakopoulos et al., 2004; Berverland, 2007), con la finalidad de evitar los indeseados comportamientos oportunistas.

En lo referente a la orientación al mercado de los socios, hay que destacar su efecto inhibidor de comportamientos oportunista. De un lado, porque esa orientación les lleva a adoptar comportamientos en el largo plazo dirigidos a satisfacer las demandas de sus clientes, y, por lo tanto, alejados del oportunismo cortoplacista. Y, de otro, porque en la medida que las cooperativas les pueden ayudar a mejorar su orientación al mercado, evitarán aquellos comportamientos que pudieran comprometer su continuidad como socios de las mismas.

Los antecedentes del oportunismo han sido analizados en el ámbito de las relaciones proveedor-cliente, pero existen escasos estudios, sobre todo empíricos, que analicen esta problemática en el ámbito de las cooperativas agroalimentarias. Tan solo encontramos referencias sobre la relación entre la heterogeneidad de los socios de las cooperativas y ciertos comportamientos oportunistas. $A$ diferencia de estos trabajos, el que aquí se presenta contempla la heterogeneidad, junto con otros factores, como antecedentes del oportunismo, lo que nos permite obtener estimaciones más robustas acerca del efecto de los mismos sobre el comportamiento oportunista de los socios. De esta forma, el presente trabajo contribuye a paliar el vacío existente en la literatura de estudios empíricos que analicen la problemática del oportunismo en el ámbito de las cooperativas agroalimentarias.

Además, consideramos que los resultados obtenidos son de interés para los responsables de dirigir las cooperativas agroalimentarias, ya que les pueden ayudar a diseñar e implementar mecanismos dirigidos a combatir los indeseados comportamientos oportunistas de sus socios. Sin embargo, el estudio no está exento de limitaciones. Por una parte, las asociadas al tamaño de la muestra que hace que los resultados obtenidos deban ser interpretados con cierta cautela para el conjunto de la población. Por otra parte, las posibles limitaciones asociadas a errores de medida, pues se ha utilizado información referente a la percepción u opinión de los dirigentes de las cooperativas sobre cada uno de 
los conceptos medidos en el cuestionario. No obstante, es necesario hacer notar que el cuestionario ha sido diseñado sobre la base de la literatura existente al respecto, con el objetivo de incurrir en el mínimo error posible.

Futuros estudios deberían utilizar muestras de mayor tamaño con las que poder explorar diferentes antecedentes del oportunismo atendiendo al tipo de cooperativa (por ejemplo, primer grado vs segundo grado), tamaño o localización de la misma. Además, tras conocer estos antecedentes en detalle, sería conveniente indagar en los posibles mecanismos de gobierno o control para gestionar el oportunismo, pues serviría de gran ayuda a los encargados de la gestión de las cooperativas.

\section{Referencias bibliográficas}

ACHROL RAVI, S. \& STERN LOUIS, W. (1988): "Environmental determinants of decision-making uncertainty in marketing channels", Journal of Marketing Research, 25, 36-50. DOI: 10.2307/3172923.

ACHROL RAVI, S. \& GUNDLACH GREGORY, T. (1999): "Legal and social safeguards against opportunism in Exchange", Journal of Retailing, 75(1), 107-123. DOI: 10.1016/S0022-4359(99)80006-2.

ANDERSON, E. (1988): "Transaction costs as determinants of opportunism in integrated and independent sales forces", Journal of Economic Behavior and Organization, 9(3), 247-264. DOI: 10.1016/0167-2681(88)90036-4.

ARCAS LARIO, N. (2000): "La relación entre las cooperativas agrarias de comercialización de primer y segundo grado. Un modelo explicativo de sus características y resultados. Universidad de Murcia. Tesis Doctoral. DOI: 10.31428/10317/755.

ARCAS LARIO, N., GARCÍA MARTíNEZ, G. \& MELIÁ MARTÍ, E. (2013): "El tamaño de las cooperativas agroalimentarias como factor de competitividad". En: Arcas Lario, N. y Hernández Espallardo, M. (Eds.), Tamaño y competitividad. Experiencias de crecimiento en las cooperativas agroalimentarias españolas (pp. 26-66). Almería, España: Cajamar Caja Rural.

ARCAS LARIO, N. \& HERNÁNDEZ ESPALLARDO, M. (2003): "Coordination and performance of Spanish second-level agricultural co-operatives: the impact of relationship characteristics", European Review of Agricultural Economics, 30(4), 487-507. DOI: 10.1093/erae/30.4.487.

BAKER WILLIAM, E. \& SINKULA JAMES, M. (1999): "The Synergistic Effect of Market Orientation and Learning Orientation on Organizational Performance", Journal of the Academy of Marketing Science, 27(4), 411-427. DOI: 10.1177/0092070399274002. 
BEVERLAND, M. (2007): "Can cooperatives brand? Exploring the interplay between cooperative structure and sustained brand marketing success", Food Policy, 32, 480-495. DOI: 10.1016/j.foodpol.2006.10.004.

BENOS, T., KALOGERAS, N., VERHEES, F.J.H.M., SERGAKI, P. \& PENNINGS, J.M.E. (2016): "Cooperatives' organizational restructuring, strategic attributes, and performance: the case of agribusiness cooperatives in Greece", Agribusiness, 32(1): 127-150. DOI: 10.1002/agr.21429.

BIJMAN, J. \& HENDRIKSE, G. (2002): "Ownership structure in agrifood chains: the marketing cooperative", American Journal of Agricultural Economics, 84(1), 104-119. DOI: 10.1111/14678276.00246 .

BIJMAN, J., ILIOPOULOS, C., POPPE, K.J., GIJSELINCKX, C., HAGEDORN, K., HANISCH, M., HENDRIKSE, G.W.J., KUHL, R., OLLILA, P., PYYKKONEN, P. \& SANGEN, G.V. (2012): Support for Farmers' Cooperatives. Final Report. Disponible en: https://ec.europa.eu/agriculture/sites/agriculture/files/external-studies/2012/support-farmers-coop/fulltext_en.pdf

BROWN JAMES, R., DEV CHEKITAN, S. \& LEE DONG, J. (2000): "Managing Marketing Channel Opportunism: The Efficacy of Alternative Governance Mechanisms", Journal of Marketing, 64(2), 51-65. DOI: 10.1509/jmkg.64.2.51.17995.

CARSON, S., MADHOK, A. \& WU, T. (2004): "The drivers of opportunism under contractual and relational governance: disentangling the effects of volatility and ambiguity in interorganizational relationships". En: Conference on Trust, Amsterdam.

CHILES, T.H. \& MCMACKIN, J.F. (1996): "Integrating variable risk preferences, trust, and transaction cost economics", Academy of Management Review, 21(1), 73-99. DOI: 10.2307/258630.

CHURCHILL, G.A. (1979): "A Paradigm for Developing Better Measures of Marketing Constructs", Journal of Marketing Research, 16, 64-73. DOI: 10.2307/3150876.

COOPERATIVAS AGRO-ALIMENTARIAS (2018): EI cooperativismo agroalimentario. Macromagnitudes del Cooperativismo Agroalimentario Español. Disponible en: http://www.agroalimentarias.coop/ficheros/doc/05708.pdf

COQUE, J. (2008): "Puntos fuertes y débiles de las cooperativas desde un concepto amplio de gobierno empresarial", REVESCO, Revista de Estudios Cooperativos, 95, 65-93.

COOK, M.L. \& ILIOPOULOS, C. (2000): "Defined property rights in collective actions: the case of US agricultural cooperatives". En: Ménard, C. (Ed.), Institutions, Contracts and Organization: Perspectives from New Institutional Economics (pp. 335-348). London: Edward Elgar Publishing.

DAS, T.K. (2006): "Strategic Alliance Temporalities and Partner Opportunism", British Journal of Management, 17, 1-21. DOI: 10.1111/j.1467-8551.2006.00482.x.

DAS, T.K. \& RAHMAN, N. (2010): "Determinants of Partner Opportunism in Strategic Alliances: A Conceptual Framework", Journal of Business and Psychology, 25, 55-74. DOI: 10.1007/s10869009-9132-2. 
EMERSON, R.M. (1962): "Power-Dependence Relations", American Sociological Review, 27(1), 31 41. DOI: $10.2307 / 2089716$.

FRAZIER, G.L. (1983): "Interorganizational Exchange Behavior in Marketing Channels: A broadened Perspective", Journal of Marketing, 47, 68-78. DOI: 10.2307/1251400.

FULTON, M. \& GIANNAKAS, K. (2001): "Organizational commitment in mixed oligopoly: agricultural cooperatives and investor-owned firms", American Journal of Agricultural Economics, 83(5), 12581265. DOI: 10.1111/0002-9092.00276.

GANESAN, S. (1994): "Determinants of Long-Term Orientation in Buyers-Seller Relationship", Journal of Marketing, 58(2), 1-19. DOI: 10.2307/1252265.

GARRIDO, I., SALAZAR, I. \& VARGAS, P. (2017): "La elección del modelo de gestión en las cooperativas españolas", REVESCO, Revista de Estudios Cooperativos, 123, 94-113. DOI: 10.5209/REVE.53251.

GREENE, W.H. (1997): Econometric Analysis, New York, Macmillan.

GRIPSRUD, G., LENVIK, G.H. \& OLSEN, N. (2000): "Influence activities in agricultural cooperatives: the impact of heterogeneity". En: The Food Sector in Transition Nordic Research, Oslo.

GUNDLACH GREGORY, T., ACHROL RAVI, S. \& MENTZER, JHON, T. (1995): "The structure of commitment in exchange", Journal of Marketing, 59(1), 78-92. DOI: 10.2307/1252016.

HANSMANN, H. (1996): The ownership of enterprise, Cambridge, The Belknap Press of Harvard University Press.

HARRIS, A., STEFANSON, B. \& FULTON, M. (1996): "New Generation Cooperatives and Cooperative Theory", Journal of Cooperatives, 11, 15-28. DOI: 10.22004/ag.econ.46188.

HART, O. \& MOORE, J. (1990): "Property rights and the nature of the firm", Journal of Political Economy, 98(6), 1119-1158. DOI: 10.1086/261729.

HAWKINS, G., WITTMANN, C. \& BEYERLEIN, M. (2008): "Antecedents and consequences of opportunism in buyer-supplier relations: Research synthesis and new frontiers", Industrial Marketing Management, 37, 895-909. DOI: 10.1016/j.indmarman.2007.05.005.

HEIDE JAN, B. (1994): "Interorganizational governance in marketing channels", Journal of Marketing, 58, 71-85. DOI: $10.2307 / 1252252$.

HEIDE JAN, B. \& JOHN, G. (1992): "Do Norms Matter in Marketing Relationships?", Journal of Marketing, 56(2), 32-44. DOI: 10.2307/1252040.

HILL CHARLES, W. (1990): "Cooperation, opportunism and the invisible hand: Implications for transaction cost theory", Academy of Management Review, 15(3), 500-513. DOI: 10.2307/258020.

HÖHLER, J. \& KÜHL, R. (2018): "Dimensions of member heterogeneity in cooperatives and their impact on organization - a literature review", Annals of Public and Cooperative Economics, 89 (4), 697712. DOI: 10.1111/apce.12177. 
ILIOPOULOS, C. \& COOK, MICHAEL, L. (1999): "The efficiency of internal resource allocation decisions in customer-owned firms: the influence costs problem". En: Actas del $3^{\text {rd }}$ Annual Conference of the International Society for New Institutional Economics (pp. 16-18), Washington D.C, September.

ILIOPOULOS, C. \& HENDRIKSE, G.W.J. (2009): "Influence costs in agribusiness cooperatives: evidence from case studies", International Studies of Management \& Organization, 11(4), 60-80. DOI: 10.2753/IMO0020-8825390404.

ILIOPOULOS, C. \& VALENTINOV, V. (2012): "Opportunism in agricultural cooperatives in Greece", Outlook on Agriculture, 41(1), 15-19. DOI: 10.5367/oa.2012.0071.

JOHN, G. (1984): "An empirical investigation of some antecedents of opportunism in a marketing channel", Journal of Marketing Research, 21(3), 278-289. DOI: 10.2307/3151604.

JOSHI ASHWIM, W. \& STUMP RODNEY, L. (1999): "Determinants of Commitment and Opportunism: Integrating and Extending Insights from Transaction Cost Analysis and Relational Exchange Theory", Canadian Journal of Administrative Sciences, 16(4), 334-352. DOI: 10.1111/j.19364490.1999.tb00693.x.

KALOGERAS, N., PENNINGS, J.M.E., VAN DER LANS, I.A., GARCÍA, P. \& VAN DIJK, G. (2009): "Understanding heterogeneous preferences of cooperative members", Agribussines, 25(9), 90111. DOI: 10.1002/agr.20187.

KYRIAKOPOULOS, K. (1998): "Agricultural cooperatives: organizing for market-orientation". En: IAMA World Congress VIII, Uruguay, 1998.

KYRIAKOPOULOS, K., MEULENBERG, M. \& NILSSON, J. (2004): "The impact of cooperative structure and firm culture on market orientation and performance", Agribusiness, 20(4), 379-396. DOI: 10.1002/agr.20021.

LAI CHI, S., LIU SHUEI, S., YANG CHIN, F., LIN HONG, W. \& TSAI HUNG, W. (2005): "Governance Mechanism of Opportunism: Integrating from Transaction Cost Analysis and Relational Exchange Theory", Taiwan Academy of Management Journal, 5(1), 1-24. DOI: 10.6295/TAMJ.2005.0501.01.

LAJARA, N. \& SERVER, R.J. (2016): "Orientación al mercado y tipología de las cooperativas agroalimentarias en base a la competitividad. Caso-estudio de las citrícolas españolas", REVESCO. Revista de Estudios Cooperativos, 121, 145-172. DOI:10.5209/rev_REVE.2016.v121.51305.

LEE DONG, J. (1998): "Developing international strategic alliances between exporters and importers: The case of Australian exporters", International Journal of Research in Marketing, 15(3), 335-348. DOI: 10.1016/S0167-8116(98)00010-X.

LUO, Y. (2007): "Are joint venture partners more opportunistic in a more volatile environment?", Strategic Management Journal, 28, 39-60. DOI: 10.1002/smj.564. 
MARCOS MATAS, G., HERNÁNDEZ ESPALLARDO, M. \& ARCAS LARIO, N. (2014): "La disposición a invertir en cooperativas agroalimentarias: el papel de la heterogeneidad y el oportunismo de los socios", Economía Agraria y Recursos Naturales, 14(1), 7-25. DOI: 10.7201/earn.2014.01.01.

OLIVER, C. (1990): "Determinants of Interorganizational Relationships: Integration and Future Directions", Academy of Management Review, 15(2), 214-65. DOI: 10.2307/258156.

ÖSTERBERG, P. \& NILSSON, J. (2009): "Members" perception of their participation in the governance of cooperatives: the key to trust and commitment in agricultural cooperatives", Agribusiness: An International Journal, 25(9), 181-197. DOI: 10.1002/agr.20200.

PARKHE, A. (1993): "Strategic alliance structuring: A game theoretic and transaction cost examination of intern cooperation", Academy of Management Journal, 36(4), 794-829. DOI: 10.2307/256759.

PING ROBERT, A.Jr. (1993): "The effects of satisfaction and structural constraints on retailer exciting, voice, loyalty, opportunism and neglect", Journal of Retailing, 69(3), 320-352. DOI: 10.1016/00224359(93)90010-G.

ROKAN AKSEL, I. \& BUVIT, A. (2003): "Inter-firm cooperation and the problem of free riding behavior: an empirical study of voluntary retail chains", Journal of Purchasing \& Supply Management, 9, 247256. DOI: 10.1016/j.pursup.2003.09.004.

SAKO, M. \& HELPER, S. (1998): "Determinants of trust in supplier relations: Evidence from the automotive industry in Japan and the United States", Journal of Economic Behavior and Organization, 34(3), 387-417. DOI: 10.1016/S0167-2681(97)00082-6.

SALAZAR TERREROS, I. \& GALVE GÓRRIZ, C. (2010): "Ejercicio de las funciones empresariales por parte de los socios en las cooperativas agrarias", REVESCO, Revista de Estudios Cooperativos, 101, 79-106.

SCHILLING MELISSA, A. \& STEENSMA KEVIN, H. (2002): "Disentangling the Theories of Firm Boundaries: A Path Model and Empirical Test", Organization Science, 13(4), 387-401. DOI: 10.1287/orsc.13.4.387.2950.

SISAY, D.T., VERHEES, F.J.H.M \& VAN TRIJP, H.C.M (2017): "The influence of market orientation on firm performance and members' livelihood in Ethiopian seed producer cooperatives", Agrekon, 56:4, 366-382, DOI: 10.1080/03031853.2017.1409126.

SKARMEAS, D., KATSIKEAS CONSTANTINE, S. \& SCHLEGELMILCH BODO, B. (2002); "Drivers of Commitment and its Impact on Performance in Cross-Cultural Buyer-Seller Relationships: The Importer's Perspective", Journal of International Business Studies, 33(4), 757-783. DOI: 10.1057/palgrave.jibs.8491043.

SWAN, J.E., BOWERS, M.R. \& RICHARDSON, L.D. (1999): "Customer Trust in the Salesperson: An integrative Review and Meta-Analysis of the Empirical Literature", Journal of Business Research, 44, 93-107. DOI: 10.1016/S0148-2963(97)00244-0. 
WALKER, G. \& WEBER, D. (1984): "A Transaction Cost approach to Make-or-Buy Decisions", Administrative Science Quarterly, 29(3), 373-391. DOI: 10.2307/2393030.

WANG, X. \& YANG, Z. (2013): "Inter-firm opportunism: a meta-analytic review and assessment of its antecedents and effect on performance", Journal of Business \& Industrial Marketing, 28, 137-146. DOI: 10.1108/08858621311295272.

WANOUS, J., REICHERS, A. \& HUDY, M. (1997): "Overall job satisfaction: how good are single-item measures", Journal of Applied Psychology 82(2): 247-252. DOI: 10.1037//0021-9010.82.2.247.

WATNE KENNETH, H. \& HEIDE JAN, B. (2000): "Opportunism in Interfirm Relationships: Forms, Outcomes and Solutions", Journal of Marketing, 64(4), 36-51. DOI: 10.1509/jmkg.64.4.36.18070.

WILLIAMSON, O.E. (1985): The Economic Institutions of Capitalism: Firms, Markets, Relational Contracting. New York, US, The Free Press. Disponible en: https://ssrn.com/abstract=1496720.

WILLIAMSOM, O. (2004): "Transaction cost economics and agriculture: an excursion". In: Huylenbroec, G., Verbeke, W., \& Lauwers, L. (Eds.), Role of Institutions in Rural Policies and Agricultural Markets (pp. 19-39), Amsterdam: Elsevier. 